\title{
Prospecção Tecnológica em Sistemas de Informação: estratégias para regularização do acesso ao patrimonio genético de uma instituição de pesquisa agropecuária
}

\author{
Technological Prospection in Information Systems: strategies for \\ regularization of access to the genetic heritage of an agricultural \\ research institution
}

\author{
Fabio Lima Cordeiro ${ }^{1}$ \\ Adelina do Socorro Serrão Belém ${ }^{2}$ \\ Alessandra Rodrigues da Silva ${ }^{3}$ \\ ${ }^{1}$ Embrapa Cerrados, Planaltina, Brasília, DF, Brasil \\ ${ }^{2}$ Embrapa Amapá, Macapá, AP, Brasil \\ ${ }^{3}$ Embrapa Sede, Brasília, DF, Brasil
}

\begin{abstract}
Resumo
A necessidade de regularização da produção técnico-científica passiva das instituições de pesquisa do país, junto ao Sistema Nacional de Gestão do Patrimônio Genético e do Conhecimento Tradicional Associado (SISGEN), está relacionada com a mudança da Lei de Acesso ao Patrimônio Genético (Lei 13.123/15) e com o Decreto que a regulariza. O objetivo deste trabalho é descrever como a prospecção tecnológica realizada por meio de um sistema de informação de uma instituição de pesquisa agropecuária pode contribuir para o atendimento ágil e seguro dos instrumentos exigidos para regularização do acesso ao patrimônio genético das atividades de pesquisa executadas pela instituição, no período anterior à promulgação do novo marco legal da biodiversidade. Os relatórios estatísticos qualitativos e quantitativos decorrentes do sistema citado foram fundamentais para o levantamento das publicações e contribuíram para a tomada de decisão dos pesquisadores responsáveis pela regularização.
\end{abstract}

Palavras-chave: Prospeção tecnológica. Acesso ao Patrimônio Genético. Sistemas de informação.

\begin{abstract}
There is a need to regularize the passive technical-scientific production of the brazilians research institutions in the National System for Management of the Genetic Heritage and Traditional Knowledge Associated (SISGEN) because of the new legal regulations in the Access to Genetic Heritage (The Brazilian Law $n^{\circ}$ 13.123/2015). The objective of this work is to describe how the technological prospection carried out through an information system of an agricultural research institution can contributes to the agile and safe service of the instruments required to regularize the access to the genetic heritage of research activities carried out by the institution, in the period before the promulgation of the new legal framework of biodiversity. The qualitative and quantitative statistical reports resulting from the information system were fundamental for prospection of publications and contributed to the decision-making of the researchers responsible for regularization.
\end{abstract}

Keywords: Technological prospecting. Access to Genetic Heritage. Information systems

Área tecnológica: Acesso ao Patrimônio Genético 


\section{Introdução}

A vida no planeta resulta de bilhões de anos de evolução, e ao longo desse período espécies surgiram e desapareceram naturalmente, como parte do processo de evolução. Entretanto as atividades humanas neste último século voltadas para a exploração dos ativos naturais deturpam e aceleram o processo de extinção de espécies e de seus ecossistemas mais do que em qualquer outro período histórico.

As perdas em biodiversidade têm aumentado de forma alarmante e estas extinções têm implicações no desenvolvimento econômico e social, além de serem uma tragédia ambiental. Quanto mais diversidade biológica, maiores são as possibilidades de descobertas no âmbito da medicina, da alimentação, do desenvolvimento econômico, e de respostas adaptativas às alterações ambientais, e por isso a variedade da vida e a utilização sustentável dos seus recursos são uma medida de segurança (ASSOCIAÇÃO O ECO, 2014a).

A preocupação com a segurança da biodiversidade do planeta tem tomado parte das agendas diplomáticas e, em 1992, durante a Conferência das Nações Unidas sobre Meio Ambiente e Desenvolvimento, mais conhecida como ECO-92, foi estabelecida a Convenção sobre a Diversidade Biológica (CDB), ratificada pelo Brasil, e que hoje é o principal fórum mundial para questões relacionadas (GROSS, et al., 2006). A CDB tem como objetivo estabelecer as normas e princípios que devem reger o uso e a proteção da diversidade biológica em cada país signatário, assegurarando a conservação da biodiversidade, o seu uso sustentável (apenas para o benefício dos seres humanos) e a justa repartição dos benefícios provenientes do uso econômico dos recursos genéticos, a um ritmo que não conduza a uma diminuição a longo prazo da diversidade biológica, respeitada a soberania de cada nação sobre o patrimônio existente em seu território (ASSOCIAÇÃO O ECO, 2014a).

Na $10^{\text {a }}$ Conferência das Partes da CDB, em 2010, definiu-se o Protocolo de Nagoia, que trata do Acesso a Recursos Genéticos e Repartição de Benefícios decorrentes da sua utilização, e que busca apresentar uma justa partilha das rendas auferidas através da exploração de processos e produtos comerciais derivados de recursos genéticos. O Protocolo é um acordo internacional suplementar à $\mathrm{CDB}$ e, por meio dele, fornecedores, como países detentores de grande biodiversidade e usuários de recursos genéticos (por exemplo, empresas farmacêuticas), desfrutarão de maior segurança jurídica e transparência em suas relações, uma vez que o novo protocolo estabelece condições mais previsíveis ao acesso a recursos genéticos e garante a repartição dos seus benefícios com quem os forneceu (ASSOCIAÇÃO O ECO, 2014b).

Durante esse contexto internacional, vigorou no Brasil a Medida Provisória $n^{\circ}$ 2.186-16, de 23 de agosto de 2001, a qual se encontrava em vigor desde 30 de junho de 2000, sobre o acesso ao patrimônio genético, a proteção e o acesso ao conhecimento tradicional associado, a repartição de benefícios e o acesso à tecnologia e transferência de tecnologia para sua conservação e utilização. Para se adequar ao objetivo do Protocolo de Nagoia, foi estabelecido, por meio da Lei $n^{\circ}$ 13.123, de 20 de maio de 2015 (BRASIL, 2015) e do Decreto no 8.772, de 11 de maio de 2016 (BRASIL, 2016), o novo marco legal da biodiversidade no país.

Pelo novo marco legal, as atividades de pesquisa executadas entre o período de vigência da Medida Provisória até a promulgação da Lei, ou seja, de 30 de junho de 2000 a 17 de novembro de 2015, devem ser regularizadas nas novas regras perante o cadastramento no Sistema Nacional de Gestão do Patrimônio Genético e do Conhecimento Tradicional Associado 
(SisGen). Estabelece ainda o marco que os cadastros devem ser realizados em até um ano após a disponibilização do sistema, o que resulta no prazo final de 6 de novembro de 2018 .

O objetivo deste trabalho é descrever como a prospecção tecnológica realizada por meio de uma sistema de informação de uma instituição de pesquisa agropecuária pode contribuir para o atendimento ágil e seguro dos instrumentos exigidos para regularização do acesso ao patrimônio genético das atividades de pesquisa executadas pela instituição, no período anterior à promulgação do novo marco legal da biodiversidade.

\section{Metodologia}

Trata-se de uma pesquisa exploratória, uma vez que, segundo Marconi e Lakatos (2003), analisa determinado tema com a finalidade de desenvolver hipóteses e aumentar a familiaridade do pesquisador com um ambiente, fato ou fenômeno para clarificar conceitos. Como reforçado por Gil (2002), o entendimento desse tipo de pesquisa é proporcionar maior familiaridade com o problema, com vistas a torná-lo mais explícito ou a constituir hipóteses, de forma que pode-se dizer que tem como objetivo principal o aprimoramento de ideias ou a descoberta de intuições. Nesse sentido, o trabalho é um estudo de caso que, ainda segundo as autoras (MARCONI; LAKATOS, 2003), é característico deste tipo de pesquisa.

Sua natureza é qualitativa uma vez que, conforme definiram Silveira e Córdova (2009), não tem preocupações apenas com a representação numérica, mas, sobretudo com a compreensão do objeto por meio de dados não métricos. Assim, o que se objetiva estudar são as características da prospecção tecnológica no Ainfo que possibilitem o atendimento de uma exigência presente no novo marco legal.

As técnicas de coleta de dados utilizadas foram: análises bibliográficas, documentais e pesquisa de campo, realizada com os bibliotecários responsáveis pela prospecção tecnólogica no sistema Ainfo. Os procedimentos adotados adequam-se à pesquisa de natureza qualitativa, conforme descrito por Gil (2002).

A pesquisa bibliográfica foi realizada junto à doutrina especializada (publicações técnico-científicas) e legislação aplicada ao novo marco legal da biodiversidade. Foram observadas as disposições do marco legal anteriormente vigente e do novo, refletindo sobre aspectos como as alterações da regulamentação e consequências, a relação das alterações para as pesquisas envolvendo patrimônio genético, e para a regularização das atividades de pesquisa executadas durante o modelo anterior.

Tabela 1 - Resumo da metodologia utilizada

$\begin{array}{ccc}\text { TIPO DE PESQuisa } & \text { TÉcnicas DE COlETA DE DAdos } & \text { Natureza } \\ \text { Exploratória } & \text { Bibliográfica } & \text { Qualitativa } \\ \end{array}$

Fonte: Elaborada pelos autores deste artigo (2018) 


\section{Resultados e Discussão}

O novo marco legal da biodiversidade é consubstanciado pela Lei n ${ }^{\circ} 13.123$, de 20 de maio de 2015 (BRASIL, 2015), e pelo Decreto no 8.772, de 11 de maio de 2016 (BRASIL, 2016). A Lei $n^{\circ} 13.123$ entrou em vigor no dia 17 de novembro de 2015, e nessa data, revogou a Medida Provisória n 2.186-16, de 2001, a qual se encontrava em vigor desde 30 de junho de 2000. O referido marco legal estabelece as regras e as condições para o acesso à amostra de patrimônio genético e ao conhecimento tradicional associado, para fins de pesquisa e desenvolvimento tecnológico (VASCONCELOS, 2016).

Tanto o novo marco legal quanto a legislação anterior procuram fornecer incentivos para a conservação e uso sustentável de recursos genéticos e, logo, da biodiversidade, sem esquecer de propor uma justa e equitativa repartição de benefícios também para as comunidades com conhecimentos tradicionais que, nesse contexto, passam a ser um dos principais beneficiados desta moldura legal, pois serão remuneradas por empresas que usufruírem dessas capacidades (ASSOCIAÇÃO O ECO, 2014b; VASCONCELOS, 2016). A importância dos marcos legais também se aplica no combate à biopirataria, pois mesmo os países que não ratificaram o Protocolo de Nagoia serão obrigados a segui-lo ao negociar com países signatários, evitando que casos como o da companhia japonesa K. K. Eyela Corporation, que obteve a patente do açaí no período de 2003 a 2007, ocorram novamente (VIALLI, 2007).

Atualmente, as atividades de pesquisa que envolvam o acesso ao patrimônio genético ou ao conhecimento tradicional associado, bem como a remessa ou o envio de amostra, devem ser cadastradas no SisGen, um sistema eletrônico do Ministério do Meio Ambiente (MMA). Criado pelo Decreto $n^{\circ} 8.772$, de 11 de maio de 2016, que regulamenta a Lei $n^{\circ} 13.123$, de 20 de maio de 2015 (Figura 1), o SisGen é um instrumento que auxilia o Conselho de Gestão do Patrimônio Genético (CGEN) na gestão do patrimônio genético e do conhecimento tradicional associado (BRASIL, 2018).

Figura 1 - Exigências da Lei no $13.123 / 2015$

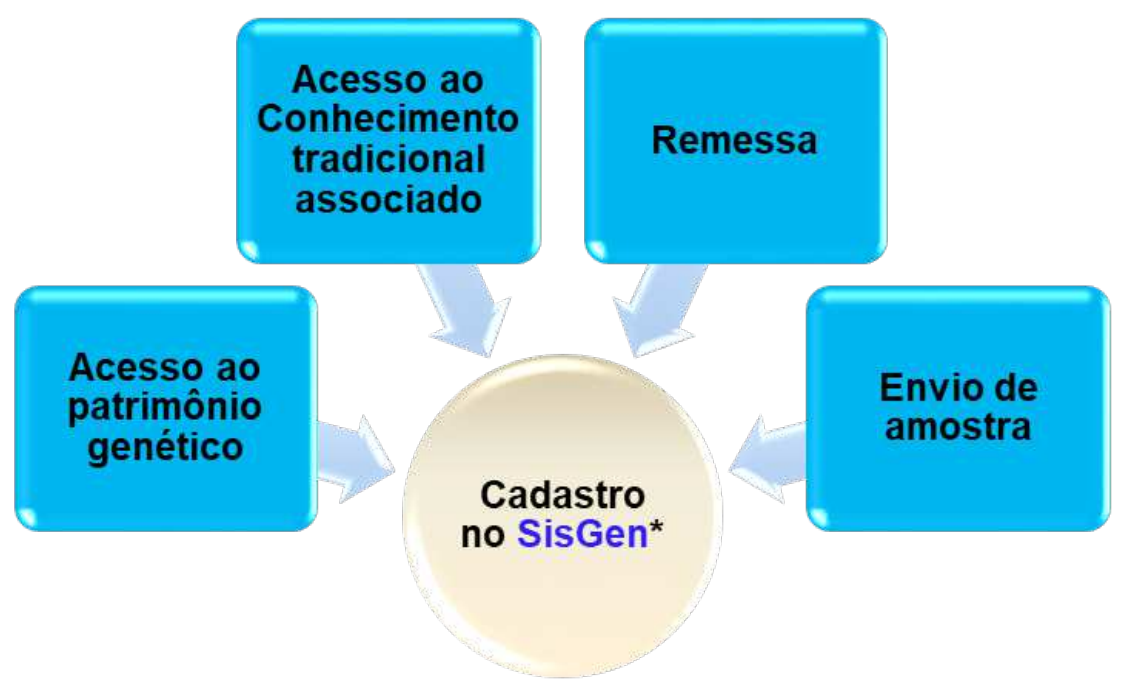

Fonte: Macêdo (2018)

No período em que a Medida Provisória (MP) n².186-16 de 2001 vigorou (de 30 de junho de 2000 a 17 de novembro de 2015), os procedimentos e as exigências legais para a coleta de 
material biológico, para o acesso à amostra de patrimônio genético e ao conhecimento tradicional associado, bem como para a repartição de benefícios oriundos da exploração de processos ou produtos desenvolvidos a partir desses acessos eram regidos pela referida MP, que era regulamentada por diversas resoluções e orientações técnicas expedidas pelo CGEN, nos termos de sua competência institucional (VASCONCELOS, 2012). Essas regulamentações alcançavam todas as atividades de pesquisa científica, bioprospecção e desenvolvimento tecnológico realizadas mediante a utilização de amostras de patrimônio genético coletadas em condições in situ, inclusive domesticadas, ou mantidas em coleções ex situ, desde que tivessem sido coletadas no território nacional, na plataforma continental ou na zona econômica exclusiva.

Segundo Vasconcelos (2012) essa MP trazia uma série de obrigações relacionadas à execução de atividades que envolvessem a utilização de amostras do patrimônio genético nativo, tais como:

a) obtenção de autorização de acesso e de remessa junto ao CGEN, ou instituição por esse credenciada, como, por exemplo, o Instituto Brasileiro do Meio Ambiente e dos Recursos Naturais Renováveis (Ibama), o Conselho Nacional de Desenvolvimento Científico e Tecnológico (CNPq) e o Instituto do Patrimônio Histórico e Artístico Nacional (Iphan); b) obtenção de anuência prévia da comunidade indígena ou local provedora do conhecimento tradicional associado; c) obtenção de anuência prévia do provedor das amostras de patrimônio genético; d) celebração de contrato de utilização do patrimônio genético e de repartição de benefícios com o provedor das amostras e/ ou do conhecimento tradicional associado; e) depósito de subamostra representativa do patrimônio genético objeto do acesso numa coleção/banco credenciado junto ao CGEN.

É oportuno mencionar, como bem coloca Vasconcelos (2012), que durante a aplicação da MP exigia-se a obtenção de autorização de acesso do CGEN, ou por instituição por esse credenciada, independentemente da data ou local da coleta da amostra de patrimônio genético. Se a amostra houvesse sido coletada na beira de uma estrada, ou comprada numa feira ou supermercado, o acesso a essa amostra para fins de pesquisa científica, bioprospecção ou desenvolvimento tecnológico deveria ser precedido de autorização (VASCONCELOS, 2012).

Sendo assim, o novo marco legal, determinou no Capítulo VIII da Lei no 13.123/2015, Das Disposições Transitórias sobre a Adequação e a Regularização de Atividades, que todas as atividades de pesquisa, bioprospecção ou desenvolvimento tecnológico executadas durante esse período de vigência da MP fossem cadastradas no SisGen, no prazo de 1 (um) ano, contado da data de sua disponibilização (BRASIL, 2015). Conforme Portaria SECEX/CGEN n 1 , de 3 de outubro de 2017, o SisGen foi implementado e disponibilizado no dia 6 de novembro de 2017 (CONSELHO DE GESTÃO DO PATRIMÔNIO GENÉTICO, 2017), o que dá o prazo de regularização dessas atividades até o dia 5 de novembro de 2018.

A regularização das atividades executadas sem as autorizações que eram exigidas pela MP no período de 30/06/00 a 17/11/2015 tem até o dia 05/11/2018 para total efetivação junto ao Sisgen, sob pena de responder a multas judiciais de valor bastante substancial variando em torno de $\mathrm{R} \$ 50.000,00$ a 500.000,00, principalmente no que diz respeito a divulgar resultados sem cadastro prévio (MACÊDO, 2018).

A Embrapa, que é uma empresa de pesquisa, desenvolvimento e inovação, possui um papel determinante na geração de conhecimentos nos setores da Agricultura e Pecuária no Brasil. 
Tem como visão ser referência mundial na geração e oferta de informações, conhecimentos e tecnologias, contribuindo para a inovação e a sustentabilidade da agricultura e segurança alimentar (EMBRAPA, 2016).

No âmbito da Embrapa, foram constituídos Grupos de Trabalhos (GT), pelas Chefias-Gerais de cada Unidade Descentralizada, para gerenciar estas regularizações exigidas pela nova Lei junto aos pesquisadores. Uma das atividades primordiais para esses GT's é realizar o inventário do passivo correspondente ao período de 30/06/2000 a 16/11/2015, referente às atividades de pesquisa.

Esse passivo é composto de atividades de pesquisa, bioprospecção ou desenvolvimento tecnológico que iniciaram, ou foram executadas, antes de 17/11/2015, sem a prévia autorização do CGEN, exigida pela Medida Provisória no 2.186-16/2001, mas para as quais o pedido de autorização foi apresentado ao CGEN, ou a instituições credenciadas (IBAMA, CNPq e IPHAN), e se encontrava em tramitação quando a Lei n ${ }^{\circ} 13.123 / 2015$ entrou em vigor (VASCONCELOS et al., 2017).

Para poder fazer o levantamento inicial deste passivo com economia de tempo, considerou-se como a melhor solução tecnológica encontrada na Embrapa a utilização do Ainfo, sistema que organiza e gere as publicações resultantes da produção técnico-científica da Empresa.

A etapa de identificação do passivo será determinada por meio de prospecção e qualificação de tecnologias. O processo de qualificação usado na Embrapa inicia-se pela etapa de monitoramento tecnológico, na qual ocorre a seguinte atividade: análise das publicações no Ainfo (OLIVEIRA; COSTA, 2014).

O Ainfo é um sistema informatizado para gestão de acervos impressos e digitais de bibliotecas que inclui todas as fases do fluxo de tratamento da informação, desde o registro das publicações, movimentações (aquisição, empréstimos, devoluções, reservas, inventário) até sua disposição aos usuários por meio de uma avançada interface de buscas. Desde sua primeira versão, em 1991, o software vem evoluindo com a implementação de novas funcionalidades requeridas por seus usuários e com atualizações tecnológicas, em especial no que se refere a novas ferramentas de software. Construído integralmente com recursos e ferramentas de software livre (Arquitetura Java EE 5: JavaServer Faces e Hibernate; Sistema Gerenciador de Banco de Dados: MySQL; Servidor Web: Tomcat), é utilizado pelo Sistema Embrapa de Bibliotecas (SEB), por organizações estaduais de pesquisa agropecuária e, também, por outras instituições públicas e privadas.

O sistema é composto de três módulos (AGROPEDIA BRASILIS, 2018):

a) Ainfo gestor: registro de informações (catalogação), movimentações de itens (empréstimos, devoluções e reservas) e relatórios (gerenciais, inventário e etiquetas);

b) Ainfo digital: módulo responsável pelo arquivamento de objetos digitais (publicações eletrônicas);

c) Ainfo consulta: buscador com interface para internet que permite consultas simples $e$ avançadas nos registros do Ainfo, incluindo o uso de vários filtros para refinamento dos resultados.

O Ainfo dispõe de um extenso conjunto de funcionalidades na gestão da informação técnico-científica. Entre elas, destaca-se a Produção Científica Institucional, objeto de estudo deste artigo. A Base Produção Científica é um subconjunto da Base Acervo. 
A inserção de registros considerados como de produção técnico-científica pode-se dar tanto via base Acervo, como via base Produção Científica. Esta última foi criada no sistema como forma de destacar o conjunto de publicações nas quais os pesquisadores da instituição constem como autores, bem como para permitir às equipes das bibliotecas que gerenciem com mais acurácia esse tipo de publicação (inserindo-os, corrigindo-os ou complementando-os).

Os campos dos registros da base Produção Científica são os mesmos da base Acervo, assim como sua operação segue as mesmas orientações destinadas à segunda (novo registro, consultar, exportar, visualizar ativados, visualizar desativados). A única exceção é que na inserção ou edição de registros, o campo Origem do material é restrito, o que impede inclusive o operador de registrar incorretamente uma publicação técnico-científica como não sendo desta categoria.

Uma vez que o campo Origem do material seja preenchido, o registro já é considerado pelo sistema como de produção científica, e os campos da aba Produção Técnico-Científica ficam aptos a serem preenchidos.

O preenchimento dos campos desta aba tem duas importantes consequências:

a) a geração de relatórios específicos para representar a produção de publicações científicas da instituição (Figura 2);

b)e a apresentação em destaque dos registros na aba Produção Científica do módulo de consulta do Ainfo (BDPA, no caso da Embrapa).

Figura 2 - Produção Científica do módulo de consulta do Ainfo



Fonte: Agropedia Brasilis (2018) 
Cada uma das Unidades da Embrapa possui um Ainfo Local, alimentado pelos profissionais, especializados em gestão da informação, que compõe o Sistema Embrapa de Bibliotecas (SEB). Além do acervo específico, os Ainfos tratam da produção técnico-científica gerada nos seus mais variados formatos (tais como artigos de periódicos, artigos em eventos, livros, capítulos de livros, cartilhas, as séries Boletim de Pesquisa e Desenvolvimento, Comunicado Técnico, Circular Técnica, Documentos, orientação de dissertações/ teses) disponibilizando-a on-line, atendendo sempre a requisitos básicos de qualidade, confiabilidade, integridade, rastreabilidade, simplicidade de conteúdo e facilidade de acesso. Esses documentos, classificados por tipo de produção técnico-científica, alimentam as bases e repositórios, gerando indicadores de produção e acesso, ajudando a traçar o perfil de atuação institucional (SOARES; ARAÚJO, 2016).

Os levantamentos estatísticos para subsidiar os GT`s responsáveis pela regularização do passivo junto ao SiSGEN, foram gerados tanto contemplando toda a produção, como individualmente por autor, em formato pdf pesquisável ou como rtf, formas finais dos relatórios para visualização, dentro de cada uma das Unidades Descentralizadas da Embrapa e posterior encaminhamento aos responsáveis pela verificação do passivo. Tudo que chega aos profissionais de gestão da informação para registro frequente e esteja de acordo com normas e padrões institucionais e respeitando licenças autorais consta na Base.

\section{Considerações Finais}

A partir do objetivo apresentado, constatou-se que o Ainfo é uma ferramenta relevante de apoio à pesquisa, uma vez que tem se configurado como fundamental no processo de regularização do acesso ao patrimônio genético das atividades de pesquisa realizadas na Embrapa, no que tange à necessidade de cadastro no SisGen, até o dia 05 de novembro de 2018. Ressalta-se o processo de alimentação e manutenção do Ainfo, pois a qualidade da informação recuperada é um dos principais fatores que têm possibilitado a realização dos levantamentos descritos, decorrente do modelo de negócio do sistema, que inclui alimentação por especialistas na área de informação e interoperabilidade com outros sistemas institucionais.

\section{Referências}

AGROPEDIA BRASILIS. Ainfo - Produção Científica. 2018. Disponível em:<https://www. agropediabrasilis.cnptia.embrapa.br/web/ainfo/producao-cientifica >. Acesso em: 22 jun. 2018.

ASSOCIAÇÃO O ECO. O que é a Convenção sobre a Diversidade Biológica. ((o) eco, Dicionário Ambiental, 22 de maio de 2014a. Disponível em: < https:/www.oeco.org.br/dicionarioambiental/28347-o-que-e-a-convencao-sobre-a-diversidade-biologica/ > . Acesso em: 7 jul. 2018.

O que é o protocolo de Nagoia. ((o) eco, Dicionário Ambiental, 30 de outubro de 2014b. Disponível em: <http://www.oeco.org.br/dicionario-ambiental/28740-o-que-e-o-protocolo-denagoia/>. Acesso em: 7 jul. 2018.

BRASIL. Decreto n 8.772, de 11 de maio de 2016. Regulamenta a Lei n ${ }^{\circ} 13.123$, de 20 de maio de 2015, que dispõe sobre o acesso ao patrimônio genético, sobre a proteção e o acesso ao conhecimento tradicional associado e sobre a repartição de benefícios para conservação e uso sustentável da biodiversidade. Diário Oficial [da] União, Brasília, DF, 12 de maio de 2016, p. 1, 
Seção 1. Disponível em: <https://www.planalto.gov.br/ccivil_03/_ato2015-2018/2016/decreto/d8772. htm >. Acesso em: 7 Jul. 2018.

. Lei no 13.123, de 20 de maio de 2015. Regulamenta o inciso II do $\S 1$ o e o $§ 40$ do art. 225 da Constituição Federal, o Artigo 1, a alínea j do Artigo 8, a alínea c do Artigo 10, o Artigo 15 e os §§ 3o e 4o do Artigo 16 da Convenção sobre Diversidade Biológica, promulgada pelo Decreto no 2.519, de 16 de março de 1998; dispõe sobre o acesso ao patrimônio genético, sobre a proteção e o acesso ao conhecimento tradicional associado e sobre a repartição de benefícios para conservação e uso sustentável da biodiversidade; revoga a Medida Provisória no 2.186-16, de 23 de agosto de 2001; e dá outras providências. Diário Oficial [da] União, Brasília, DF, 21 de maio de 2015, p. 1, Seção 1. Disponível em: <http://www.planalto.gov.br/ccivil_03/_ato2015-2018/2015/lei/113123. htm >. Acesso em: 7 jul. 2018.

Ministério do Meio Ambiente. Sisgen. 2018. Disponível em: http://www.mma.gov.br/ patrimonio-genetico/conselho-de-gestao-do-patrimonio-genetico/sis-gen. Acesso em: 7 jul. 2018.

CONSELHO DE GESTÃO DO PATRIMÔNIO GENÉTICO. Secretaria Executiva. Portaria $n^{\circ} 1$, de 3 de outubro de 2017. Implementa e disponibiliza o Sistema Nacional de Gestão do Patrimônio Genético e do Conhecimento Tradicional Associado - SisGen a partir de 6 de novembro de 2017. Diário Oficial [da] União, Brasília, DF, 13 de outubro de 2017, p. 78, Seção 1. Disponível em: <http://www.mma.gov.br/images/arquivo/80043/Portaria_001_CGEN__DOU1_13_10_p78.pdf > . Acesso em: 6 jul. 2018.

EMBRAPA. Missão, visão e valores. 2016. Disponível em: < https://www.embrapa. br/missaovisao-e-valores >. Acesso em: 22 jun. 2018.

GIL, A. C. Como elaborar projetos de pesquisa. 4. ed. São Paulo: Atlas, 2002. 176 p. Disponível em: <https://professores.faccat.br/moodle/pluginfile.php/13410/mod_resource/content/1/como_ elaborar_projeto_de_pesquisa_-_antonio_carlos_gil.pdf > . Acesso em: 27 maio 2018.

GROSS, T.; JOHNSTON, S.; BARBER, C. V. A convenção sobre diversidade biológica: entendendo e influenciando o processo: um guia para entender e participar efetivamente da oitava Reunião da Conferência das Partes da Convenção sobre Diversidade Biológica. Brasília, DF: MMA, Secretaria de Biodiversidade e Florestas, 2006. 76 p. Disponível em: <http://www.mma.gov.br/ estruturas/sbf_chm_rbbio/_arquivos/entendendo\%20e\%20influenciando\%20a\%20CDB.pdf $>$. Acesso em: 7 jul. 2018.

MACÊDO, F. S. Novo marco legal da biodiversidade - Lei no 13.123/2015. Brasília, DF: Embrapa. Secretaria de Inovação e Negócios, 2018. 45 p.

MARCONI, M. A.; LAKATOS, E. M. Fundamentos da Metodologia Científica. 5. ed. São Paulo: Atlas, 2003. 315 p. Disponível em: < https://docente.ifrn.edu.br/olivianeta/disciplinas/copy_of_ historia-i/historia-ii/china-e-india >. Acesso em: 5 jul. 2018.

OLIVEIRA, R. C. de; COSTA, T. C. P. das N. Prospecção tecnológica aplicada à pesquisa agropecuária na Embrapa Amazônia Oriental. Belém, PA: Embrapa Amazônia Oriental, 2014. 23 p. (Embrapa Amazônia Oriental. Documentos, 399). Disponível em: <http://ainfo.cnptia. embrapa.br/digital/bitstream/item/100184/1/DOC-399.pdf>. Acesso em: 7 jul. 2018.

SILVEIRA, D. T.; CÓRDOVA, F. P. A pesquisa científica. In: GERHARDT, T.; SILVEIRA, D. T. (Org.). Métodos de Pesquisa. Porto Alegre: Ufrgs, 2009. Cap. 2. p. 34-42. Disponível em: <http://http:// meiradarocha.jor.br/news/tcc/files/2017/12/Gerhardt-e-Silveira.-M\%C3\%A9todos-de-Pesquisa-EADUFRGS.pdf > . Acesso em: 27 maio 2018. 
SOARES, E. A. de A.; ARAUJO, C. R. M. de. A produção técnico-científica da Embrapa Agroindústria de Alimentos à luz do sistema AINFO e dos repositórios institucionais: uma análise quantitativa do período de 2012 a 2015. Rio de Janeiro: Embrapa Agroindústria de Alimentos, 2016. 15 p. (Embrapa Agroindústria de Alimentos. Documentos, 125). Disponível em: <http://ainfo.cnptia.embrapa.br/digital/bitstream/item/157536/1/DOC-125-producao-CTAA1.pdf > . Acesso em: 22 jun. 2018.

VASCONCELOS, R. M. de (Ed.). Marcos regulatórios aplicáveis às atividades de pesquisa e desenvolvimento. Brasília, DF: Embrapa, 2016. 182 p. il. Disponível em: <http://ainfo.cnptia. embrapa.br/digital/bitstream/item/157337/1/Marcos-regulatorios-aplicaveis-as-atividades-depesquisa-e-desenvolvimento-2016.pdf> . Acesso em: 22 jun. 2018.

Marco regulatório sobre acesso à amostra de patrimônio genético nativo e acesso ao conhecimento tradicional associado. Brasília, DF: Embrapa Informação Tecnológica, 2012 58 p. Disponível em: < https:/ainfo.cnptia.embrapa.br/digital/bitstream/item/91361/1/MarcoRegulatorio-sobre-Acesso.PDF>. Acesso em: 7 jul. 2018.

VASCONCELOS, R. M. de et al. Manual da Embrapa para cadastramento de atividades no SisGen. Brasília, DF: Embrapa, 2017. 96 p. Disponível em: < http://ainfo.cnptia.embrapa.br/digital/ bitstream/item/174957/1/MANUAL-DA-EMBRAPA-PARA-CADASTRAMENTO-DE-ATIVIDADES-NOSISGEN.pdf > . Acesso em: 7 jul. 2018.

VIALLI, A. Brasil recupera de japoneses registro da marca 'açaî'. O Estado de São Paulo, 21/02/2007, Economia, p. B4. Disponível em: <http://www2.senado.leg.br/bdsf/handle/id/325622>. Acesso em: 27 maio 2018.

\section{Sobre os autores}

\section{Fabio Lima Cordeiro}

E-mail: agaciel@hotmail.com

Graduado em Biblioteconomia (UnB). Mestrando em Propriedade Intelectual e Transferência de Tecnologia para a Inovação (PROFNIT/UnB).

Endereço profissional: Embrapa Cerrados - Rodovia BR 020, Km 18 - Zona Rural- Planaltina - CEP: 73310970 - Brasília, DF.

\section{Adelina do Socorro Serrão Belém}

E-mail: adelina.belem@embrapa.br

Graduada em Biblioteconomia (UFPA), Mestranda em Propriedade Intelectual e Transferência de Tecnologia para a Inovação (PROFNIT/Unifap).

Endereço profissional: Embrapa Amapá - Rodovia Juscelino Kubitschek, Km 5, nº 2.600 - Universidade - CEP: 68903-419 - Macapá, AP.

\section{Alessandra Rodrigues da Silva}

E-mail: alessandra.silva@embrapa.br

Graduada em Biblioteconomia (UFMG). Doutora em Ciência da Informação (UnB).

Endereço profissional: Embrapa Sede - Parque Estação Biológica - PqEB s/nº. CEP 70770-901 - Brasília, DF. 